\title{
Capitolo III De cane: P.Oxy. XI 1404
}

Puntare con avidità a più di quanto già non si possegga può essere deleterio e può far perdere anche quello che si è precedentemente conquistato con fatica: la favola del cane e del riflesso della carne che trascina in bocca è un ammonimento a chi, preso da ostinata cupidigia, non si accontenti di quanto ha. D'altro canto, in tutta la tradizione favolistica che lo vede protagonista, il cane è $\pi \lambda \varepsilon$ ovékтns per eccellenza, avido di guadagno e mai contento di quanto gode ${ }^{1}$.

Il tema è noto a partire da Teognide, tra i cui versi si legge di un cane che, attraversando un ruscello, perde tutto quello che ha $(347-348)^{2}$. Il nucleo narrativo originario, però, si fonde ad un motivo cinico per eccellenza, quello dell'avidità punita ${ }^{3}$, e diventa insegnamento diffuso tra Occidente ed Oriente, filtrato - a partire da un originario nucleo esopico in greco che ebbe grossa fortuna fino all'Occidente medievale - in lingua latina e circolato nei milieux intellettuali (e, forse, scolastici) della Ossirinco tardoantica, quando qualcuno, ellenofono o latinofono che fosse, leggeva e rifletteva sulla favola del P.Oxy. XI 1404.

\section{III.1 De cane}

Trovato un pezzo di carne, un cane si accingeva ad attraversare un fiume stringendolo nella bocca; quando, però, vide riflessa nell'acqua l'illusoria immagine di quel pezzo di carne, credette fosse un altro. L'esiguità del frammento ossirinchita si arresta a questo punto della storia, ma l'epilogo è noto, benché

Secolo: III d.C.

Provenienza: Oxyrhynchus (Bahnasa)

Edizioni: B.P. Grenfell, A.S. Hunt, 1915 (P.Oxy. XI 1404)

Repertori: CPL 38; CLA XI 1667; LDAB 136; MP³ 3010

Conservazione: Wellesley (Massachusetts), Wellesley College

Documento esaminato attraverso le riproduzioni fotografiche digitali

1 Per una ricostruzione degli elementi narrativi della favola in analisi, per una rassegna sulle fonti e sulla relativa bibliografia, e per un possibile stemma ci si limita qui a rinviare a Rodríguez Adrados 2003, 174-178 (H. 136).

$2 \mathrm{Su}$ questi versi e sull'immagine teognidea ci si limita qui a rinviare a Napolitano 1996.

$3 \mathrm{Su}$ questo tema cinico e sulla sua presenza in ulteriori favole si confronti Rodríguez Adrados 1999a, 614; d'altro canto, accontentarsi di quanto si ha è tema che torna spesso anche all'interno delle favole di Babrio, a proposito della quali si vedano le osservazioni di Morgan 2007, 378-379. 
rimaneggiato nelle differenti versioni della favola: il cane perse il suo pezzo di carne per aver tentato di afferrarne un altro che era soltanto frutto di illusione.

Tra la raccolta esopica e Babrio qualche differenza si registra nella misura in cui la prima sottolinea la volontà del cane di privare del suo pezzo di carne l'altro (supposto) cane che vedeva nel riflesso dell'acqua, mentre il secondo si concentra soltanto su quanto stimolava il desiderio del cane, sulla carne ${ }^{4}$; nell'una e nell'altra versione della favola, però, il pezzo di carne riflesso nell'acqua attira l'attenzione del cane perché gli sembra più grosso di quello che stringe tra le labbra, e la spinta ad afferrarlo nasce dalla volontà di appropriarsi di qualcosa di superiore rispetto a quanto si ha. Il desiderio di strappare via una praeda ad un altro cane è parimenti enfatizzato nei senari di Fedro, il cui insegnamento sarà proprio quello di frenare il desiderio di tutto quanto sia altrui; in Fedro il desiderio del cane nasce dal voler afferrare non qualcosa di più grosso che il suo stesso pezzo di carne, ma qualsiasi altra cosa appartenga ad un altro 5 .

La favola del cane e del pezzo di carne entrò e rimase a lungo nel campionario di scuola: una sua rielaborazione è raccolta nell'opuscoletto favolistico di Aftonio ${ }^{6}$, e prima ancora, illustrando quell'esercizio progimnasmatico che è la favola stessa, Elio Teone usa questo racconto come esempio per illustrare al suo discente come sia possibile rimodulare ed esercitarsi sulla favola aggiungendole una morale, nello sforzo di farle calzare un enunciato gnomico che sintetizzi il suo insegnamento e valore ${ }^{7}$.

La favola entrò anche nel nucleo accorpato agli Hermeneumata Pseudodositheana, all'interno di una sequenza di narrazioni che esortano parimenti ad un

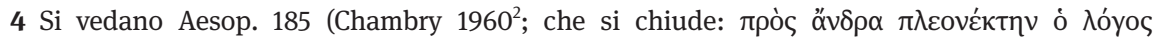

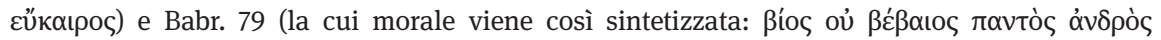

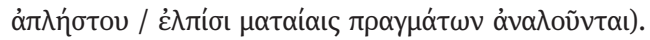

5 Phaedr. 1, 4, sulla quale si confrontino le annotazioni di commento di Oberg 2000, 48-49 e, più recentemente, le osservazioni di Gärtner 2015, 108-114.

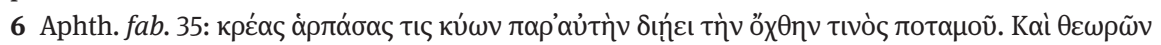

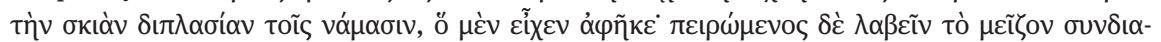

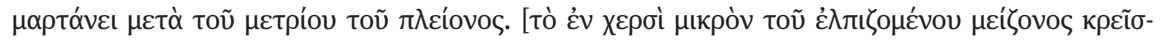
бov]. Sulle favole della raccolta di Aftonio e per ulteriori rinvii bibliografici si confronti supra.

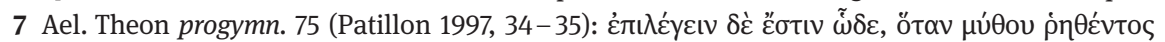

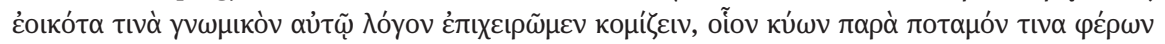

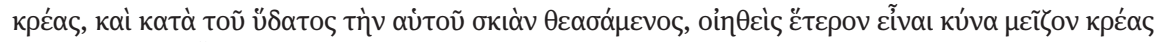

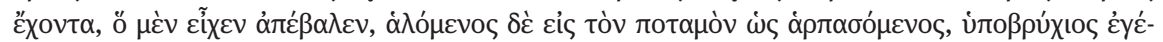

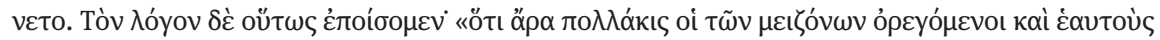

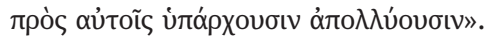


atteggiamento morigerato ${ }^{8}$. Le due recensioni delle favole degli Hermeneumata Pseudodositheana, quella, cioè, nota dai Leidensia e quella del Fragmentum Parisinum, benché presentino lo stesso sviluppo narrativo e la stessa massima morale, non coincidono nella forma e sono evidente espressione di 'deviazioni' a partire da una stessa base testuale'; che questo testo-base in latino avesse punti di contatto con quello del P.Oxy. XI 1404 è un'ipotesi che si muove in parallelo con l'analisi stessa delle sequenze e delle forme che assume lo sviluppo narrativo.

\section{III.2 P.Oxy. XI 1404}

La favola è ricopiata al verso papirologico del frammento, evidentemente appartenente ad un rotolo e che era stato utilizzato al recto, in perpendicolare alle fibre, per ricopiare dei conti in greco in una scrittura datata alla seconda metà del II secolo ${ }^{10}$. Le dimensioni del frammento $(10.6 \times 5.9 \mathrm{~cm})$ sono troppo esigue perché si possa tentare una ricostruzione delle dimensioni del rotolo originario ${ }^{11}$.

8 In questi termini ci si è recentemente espressi a proposito della presenza della favola del cane e del pezzo di carne all'interno degli Hermeneumata: «la stessa favola è presente, in redazione greca e latina, negli Hermeneumata Pseudodositheana, ma le due versioni in questo caso rinviano al testo della raccolta esopica e a Babrio» (Pugliarello 2014, 83). Su questa conclusione sarà opportuno ritornare, innanzitutto tenendo presente che le due recensioni della stessa favola non sono concordi in tutti i dettagli testuali. D'altro canto, più problematico era stato l'inquadramento di Getzlaff 1907, 10 -12, dove la versione della favola nota dagli Hermeneumata viene messa in parallelo con quella del Romulus, individuando punti di contatto ed un'origine comune, lontana dalla versione della favola nota da Babrio. La questione dei punti di contatto tra le varie recensioni degli Hermeneumata e quelle del Romulus è molto complessa, tanto più che in alcune di queste è evidente l'ingresso di elementi dichiaratamente fedriani (originari? o frutto di una stratificazione a partire da un nucleo testuale preesistente?).

9 Una nuova edizione tradotta e commentata di questa favola nella sua recensione parigina e limitatamente al testo latino è in Mordeglia 2015, 178-180.

10 È stata anche avanzata l'ipotesi che queste quattro linee in greco fossero parte di un esercizio matematico; in merito si confronti, anche per ulteriori rinvii bibliografici, Legras 1996, 75. 11 La presenza di uno spazio vacuo di $2.5 \mathrm{~cm}$ al di sotto dell'ultima linea latina al verso ha suggerito la possibilità che il testo fosse stato interrotto; si confronti P.Oxy. XI 1404, 247: «there is a blank space of $2.5 \mathrm{~cm}$. after 1.4 and no trace of writing below, which would be expected to be visibile if other lines followed immediately». Non si può escludere, però, che si trattasse della sezione inferiore del rotolo e che il vacuo rappresenti una parte del margine. 
La scrittura latina è una corsiva antica inclinata a destra, databile al III secolo e frutto dell'operazione di uno scriba esperto ${ }^{12}$, uno scriba che non nasconde una marcata tendenza cancelleresca identificabile con quella di una mano burocratica ${ }^{13}$.

Una riflessione si impone sulla necessaria distinzione tra lo scriba che ha copiato il testo sul rotolo ed il compilatore della parafrasi favolistica in sé. Le due figure, infatti, sono state confuse ed identificate: se ne è parlato come di un ellenofono alle prese con l'apprendimento del latino per accedere alle frange dell'amministrazione romana ${ }^{14}$, e come di un principiante che non doveva avere il latino come lingua madre e che, al contrario, ne aveva una scarsa conoscenza in relazione a grammatica e a pronuncia ${ }^{15}$.

L'abilità scrittoria della mano che ha ricopiato il testo del P.Oxy. XI 1404 fa, però, piuttosto riflettere sulla possibilità che lo scriba debba essere distinto dal compilatore della parafrasi: uno scriba ellenofono, abile e disinvolto nella pratica della scrittura latina, potrebbe aver attinto il suo testo da un antigrafo. In questa prospettiva anche le imperfezioni scrittorie potrebbero essere differentemente inquadrate, perché non sono necessariamente ascrivibili al compilatore della parafrasi latina quanto piuttosto al copista, tanto più se ellenofono.

\section{III.3 II P.0xy. XI 1404 e la tradizione esopica}

Che il testo veicolato dal P.Oxy. XI 1404 fosse la parafrasi della favola del cane che stringeva tra le labbra un pezzo di carne è cosa messa in rilievo fin dall'editio princeps, pubblicata nella serie dei papiri di Ossirinco da Grenfell ed Hunt nel 1915; che, invece, si trattasse della parafrasi della favola di Fedro $(1,4)$ è opinione che è andata consolidandosi nella storia degli studi, con la conseguenza che il papiro sarebbe espressione non soltanto di una fruizione scolastica di Fedro ma anche della sua circolazione negli ambienti culturali e formativi

12 Si veda la descrizione paleografica di Ammirati 2015, 39, dove si troveranno anche ulteriori rinvii bibliografici.

13 Cavallo 2008, 161.

14 Si veda Legras 1996, 75: «cette paraphrase latine d'une fable latine doit émaner de ces milieux hellénophones, qui apprennent le latin à leur enfants pour leur permettre d'accéder aux métiers de l'administration romaine».

15 Legras 1996, 75-76 afferma, infatti, che «notre jeune latiniste ne semble pas en effet avoir le latin pour langue maternelle: il n'en maîtrise ni la grammaire ni la prononciation», confondendo imperfezioni che potrebbero essere di scriba con quelle del compilatore stesso (e, talora, addirittura di maestri incompetenti; si confronti, nello specifico, Legras 1996, 63). 
dell'Egitto ellenofono di III secolo. Si recupererebbe, addirittura, in tal modo il più antico manoscritto fedriano superstite, nella misura in cui bisognasse immaginare i senari di Fedro come modello della parafrasi trasmessa dal papiro. Benché, infatti, il tema favolistico sia noto fin dal corpus esopico e dalla raccolta di Babrio, si è creduto che ad ispirare la parafrasi anonima del papiro siano stati piuttosto i senari di Fedro ${ }^{16}$, cosa questa che consacrerebbe il papiro ad un'importanza focale nella ricostruzione della circolazione dell'auctor della favola latina come modello letterario e scolastico ${ }^{17}$.

Il fatto che il cane non costeggi il fiume, l'aver sorvolato sul furto della carne, e l'assenza della scena in cui il cane vede scivolarsi di bocca il suo pezzo di carne sono stati interpretati come tratti comuni alla narrazione di Fedro e a quella veicolata dal frammento ossirinchita ${ }^{18}$. Si tratta, però, di tre punti che possono essere anche interpretati differentemente che nella prospettiva fedriana.

C'è, innanzitutto, un elemento che segna nella favola un'incongruenza (e, indubbiamente, un'innovazione) tutta di Fedro: il cane viene ritratto mentre nuota nel fiume (1, 4, 2: canis per flumen carnem cum ferret natans) e, se immerso nell'acqua, è improbabile che abbia potuto vedere riflessa sulla superficie dell'acqua (1, 4, 3: lympharum in speculo) la propria immagine; di questo si rese conto la maggior parte dei continuatori della favola di Fedro, e l'idea di un guado o piuttosto quella di un ponte si introdusse nella narrazione ${ }^{19}$. Se il compilatore della parafrasi del P.Oxy. XI 1404 avesse avuto dinanzi i senari di Fedro e se fosse stato un discente alle prime armi che avrebbe pedissequamente seguito il modello, probabilmente avrebbe ripetuto l'errore fedriano e ritratto il cane mentre nuotava; d'altro canto, però, avrebbe potuto anche 'correggere' Fedro, non ri-

16 Si confronti, da ultima, in questa prospettiva Pugliarello 2014, 82-83. In Fernández Delgado 2006 si parla di questo frammento ossirinchita come di una «paráfrasis relativamente temprana de Fedro I 4» (35), probabilmente anche sulla scia delle osservazioni di Rodríguez Adrados 1999b, 9.

17 In termini forse troppo entusiastici si esprime Fernández Delgado 2006, 35: «no solo es este el papiro escolar latino más antiguo, sino también el segundo testimonio más antiguo que poseemos de la obra de Fedro, solamente superado en antigüedad por el testimonio siguiente, una paráfrasis fedriana en griego»; la parafrasi in questione («el segundo testimonio») sarebbe quella greca del P.Köln II 64, a proposito della quale si confrontino le osservazioni supra.

18 In merito si vedano, infatti, Legras 1996, 75 n. 135 e, ad un decennio di distanza, la ripresa di Fernández Delgado 2006, 35.

19 A proposito di questa incongruenza fedriana e di come i continuatori di Fedro l'abbiano filtrata è opportuno rinviare alle osservazioni di Navone 1987, formulate in seno ad uno studio che analizza la fortuna nelle raccolte mediolatine e romanze della favola fedriana in questione (per l'introduzione del guado o del ponte si veda, in particolare, 191-193). Sulla fortuna di questa favola di Fedro fino all'età contemporanea si confrontino anche le osservazioni e le versioni raccolte da Bertini 2009b. 
petere la sua ingenuità - per quanto la verosimiglianza non sia necessariamente un elemento distintivo della tradizione favolistica - e dipingere, più genericamente, un cane che non nuotava nel fiume ma che piuttosto lo attraversava. Se fosse così, però, il transire della parafrasi ossirinchita sembra più vicino

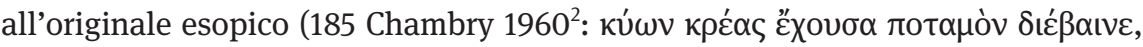

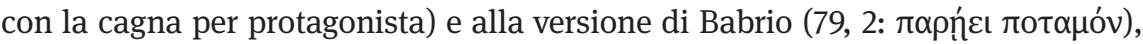
nonché alla versione della favola di quel manuale scolastico bilingue che sono gli Hermeneumata Pseudodositheana, identica nelle due recensioni note e più vicine all'originale esopico che ai versi di Babrio su questo dettaglio narrativo

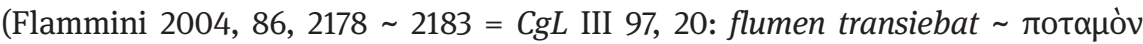

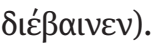

Quanto, invece, all'assenza nel frammento papiraceo della scena in cui il pezzo di carne scivola via dalla bocca del cane, benché non si possa categoricamente escludere che fosse anche nel manoscritto originario, il primo e più naturale impedimento ad esserne certi è la rottura del pezzo che avrebbe così potuto causare l'interruzione della narrazione.

Indipendentemente dal fatto che aver parafrasato una favola può aver generato delle variazioni nel lessico impiegato dal parafraste stesso, ci sono, però, degli elementi singolari della narrazione nota dal frammento ossirinchita. Se, infatti, la tradizione è bipartita tra l'attribuire al cane un furto del pezzo di carne - si veda la versione esopica, quella di Babrio, e poi Aftonio e gli Hermeneumata, con forme verbali oscillanti tra $\kappa \lambda \dot{\varepsilon} \pi \tau \omega$, $\alpha \rho \kappa \alpha ́ \alpha \omega$ e rapio - e tacere completamente sul dettaglio della provenienza della carne stessa - è tale il silenzio di Fedro e di Elio Teone -, la versione ossirinchita attribuisce piuttosto al cane l'aver trovato (invenit) il suo pezzo di carne.

Più antico testimone manoscritto della parafrasi scolastica in latino di una favola, quella ossirinchita ha, d'altro canto, analogie stringenti con la versione della favola degli Hermeneumata e con quella del Romulus $(6)^{20}$ : il segmento che ritrae l'attraversamento del fiume si ripropone identico tramite l'uso del verbo transire (e tramite la stessa confusione del verbo, come fosse di quarta coniugazione e non composto di eo) e del riflesso del pezzo di carne si parla sempre come di un'umbra. Si tratta di denominatori comuni che segnano un decisivo allontanamento dalla favola di Fedro e che, allo stesso tempo, costituiscono elementi chiave non soltanto in termini stricto sensu lessicali ma anche in termini concettuali; si tratta, soprattutto, di denominatori comuni fra la tradizione

20 Le versioni latine della favola del cane e della fetta di carne, da Fedro fino al Romulus, sono date in parallelo infra. Già Della Corte 1966, 542-543 aveva messo in luce le analogie tra la parafrasi ossinchita e la favola degli Hermeneumata. 
latina della favola ossirinchita, quella degli Hermeneumata e, poi, del Romulus, da un lato, e quella esopica e babriana (insomma, in greco), dall'altra ${ }^{21}$.

Nella parafrasi del frammento ossirinchita scompare - almeno limitatamente alle linee superstiti - un altro tema esopico, quello, cioè, che la fetta di carne riflessa nell'acqua desse al cane l'impressione di essere 'più grande'

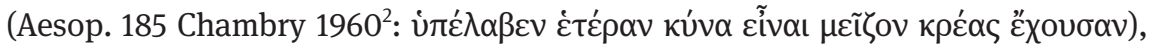

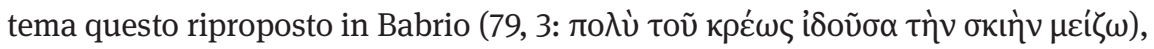
nelle versioni scolastiche di Elio Teone ed Aftonio ${ }^{22}$, nella recensione leidense degli Hermeneumata (Flammini 2004, 86, 2179 2184: ن் suspicatus est maiorem esse) e nella recensio vetus del Romulus (maiorem suspicatus est), ma non nel Fragmentum Parisinum (CgL III 97, 22-23: aestimans

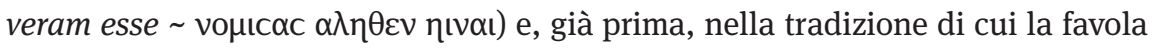
di Fedro è un'espressione.

Il P.Oxy. XI 1404, inoltre, fa riferimento al riflesso del solo pezzo di carne, e non a quello del cane stesso: scompare il tema dell'alterità e della volontà di privare un altro di qualcosa per renderlo proprio, tema che aveva avuto voce nei senari di Fedro e ancora prima nella versione esopica e, poi, nei progimnasmi di Elio Teone, ma non in Babrio e nelle rielaborazioni scolastiche degli Hermeneumata Pseudodositheana e di Aftonio, prima ancora che nella recensio vetus del Romulus. Questa bipartizione ha un esito anche in una bipartizione dell'insegnamento della favola stessa: se, da un lato, l'assenza del tema dell'altro genera un insegnamento tale per cui è opportuno accontentarsi di quanto si possiede piuttosto di tentare di ottenere cose incerte, dall'altro, sottolineare la volontà di privare un altro cane (quello frutto dell'illusorio riflesso dell'acqua) del suo pezzo di carne è di insegnamento perché il desiderio delle cose altrui può indurre a perdere anche le proprie. Quale fosse l'insegnamento che il parafraste della favola nota dal papiro ossirinchita volesse trasmettere non è noto dalle linee superstiti, ma, dal momento che il riferimento è al riflesso della sola fetta di carne, si può dedurre che la morale doveva essere quella per cui è opportuno accontentarsi di quanto di certo si possegga.

21 Sia nella raccolta esopica che in quella di Babrio si fa riferimento ad una okı́ per il riflesso

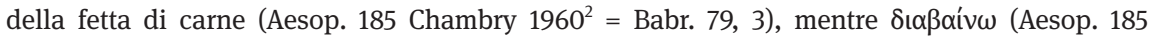
Chambry 1960²) е тó $\rho \varepsilon \mu$ (Babr. 79, 2) sono rispettivamente i verbi che sintetizzano l'attraversamento del fiume. Analizzando la favola del Fragmentum Parisinum, Mordeglia 2015, 179 ha rintracciato nel particolare narrativo del riflesso della carne piuttosto che quella di un secondo cane un elemento per il quale la tradizione degli Hermeneumata divergerebbe da quella fedriana; ma il tema è antico e già di tradizione esopica.

22 Si confronti supra il testo di riferimento. 
Quella sui rapporti tra il testo del P.Oxy. XI 1404 ed il resto della tradizione è, dunque, una riflessione che si impone, ma alla quale dare una risposta univoca è complesso, se non altro perché resta di fatto che quella della favola è una tradizione troppo flessibile e sfuggente per essere oggetto di assolutizzazioni di sorta. I punti di contatto con la tradizione degli Hermeneumata Pseudodositheana e con quella nota dalla recensio vetus del Romulus sono, però, allo stesso tempo, troppo evidenti per essere trascurati e non interrogarsi sul fatto che, probabilmente, una stessa tradizione 'esopica' dovette trovare voce in questi vari testi, o in modo indipendente l'uno dall'altro, tra Oriente ed Occidente, o attraverso rapporti di filiazione, derivazione, o influenza reciproca impossibili a ricostruirsi; d'altro canto, anche la morale tutta fedriana della recensio vetus del Romulus potrebbe essere aggiunta posteriore, tanto più che l'insegnamento di non desiderare le cose altrui male si adegua alla favoletta trasmessa, in cui non c'è il riferimento ad un 'altro cane' (riflesso dall'acqua) e al desiderio di sottrargli quanto proprio ${ }^{23}$.

La frammentarietà del testo veicolato dal testimone ossirinchita lascia aperto un ulteriore interrogativo, che, cioè potesse trattarsi di un testo sviluppato in modo analogo agli altri testimoni orientali di favole bilingui latino-greche, con la versione latina a precedere quella greca, per cui si sarebbe persa non soltanto la morale della favola ma anche una sua possibile resa al greco, antecedente diretto di una tradizione che, rimodellata e stratificata nel tempo (e nello spazio), è approdata alle recensioni medievali (ed occidentali) degli Hermeneumata Pseudodositheana ${ }^{24}$.

canis carnem inv[e]nit et flu-

-men t<rıansi\{e\}bat, deinde cum in

aquam vidisset umbram car-

nis existima[v]it altera $\triangleleft \mathrm{m}>$

23 È in Getzlaff 1907, 10 -12 che si traccia un bilancio delle analogie tra il testo degli Hermeneumata e quello del Romulus; naturalmente, Getzlaff non conosceva ancora il frammento ossirinchita.

24 A proposito del papiro si legge in Mordeglia 2015, 180: «questa testimonianza prova, se non la diffusione diretta delle favole fedriane nelle provincie orientali dell'impero romano in età tardoantica, impossibile da dimostrare dinanzi a una tradizione tanto variegata e condizionata da una componente orale scarsamente tracciabile, l'ampia fortuna di questo tema favolistico in lingua latina anche in zone periferiche in un periodo antecedente alla stesura degli Hermeneumata. Nonostante una somiglianza linguistica oltre che contenutistica, non ci sono tuttavia elementi sufficienti per ipotizzare un rapporto di derivazione diretto tra le due versioni della favola». Resta, però, incerto se questi papiri diano voce ad una tradizione approdata a quella degli Hermeneumata, la cui 'stesura' (nella forma fissata dalla tradizione manoscritta medievale) è frutto di una stratificazione che aveva avuto inizio dall'antichità; in merito, si confronti supra. 
1 INV[E]NIT: della prima I è visibile la sola estremità inferiore, di N resta parte del tratto verticale, mentre di v la sezione curva del modulo; quanto alla seconda $\mathrm{N}$ resta l'estremità superiore del tratto obliquo con il caratteristico empattement || 4 -[v]IT: di I resta parte del tratto, mentre T è inequivocabile e manchevole soltanto di una parte del tratto orizzontale; di L, poco dopo, si vede solo l'apicatura superiore del tratto verticale, mentre di $\mathrm{R}$ resta esclusivamente parte del tratto verticale

2 tansiebat pap.: transibat legendum

11. 1-2: canis carnem inv[e]nit et flu- | -men tırsansi\{e\}bat

È stata recentemente richiamata l'attenzione su una possibile analogia tra questa espressione del papiro e Phaedr. 1, 4, 2: canis per flumen carnem cum ferret natans ${ }^{25}$, benché più radicato sembri il parallelo con l'esordio della favola negli Hermeneumata Pseudodositheana e, in particolare, con la recensione nota dal Fragmentum Parisinum, dove ugualmente viene instaurato un rapporto di coordinazione tra l'aver reperito - si voglia per sorte, si voglia per un furto - la carne e l'attraversare il fiume.

Comune alla favola degli Hermeneumata è anche l'aver flesso il verbo transire come uno di quarta coniugazione e non come un composto di eo: la possibilità che si tratti di un'imperfezione già di antigrafo (se non addirittura di autore) sembra marciare di pari passo con quella che la comunanza di errore costituisca un'importante prova testuale a corroborare il legame di tradizione tra la parafrasi del papiro ossirinchita e quella degli Hermeneumata.

La giustapposizione dell'aoristo con l'imperfetto del testo del P.Oxy. XI 1404 è stata letta da Della Corte come uno degli elementi che caratterizzerebbe il «miglior latino del papiro» ${ }^{26}$ rispetto a quello degli Hermeneumata Pseudodositheana.

11. 2-3: deinde cum in | aquam vidisset umbram car- | -nis

Questa dell'anonimo compilatore ossirinchita è stata interpretata recentemente come una parafrasi di Phaedr. 1, 4, 3: lympharum in speculo vidit simulacrum suum $^{27}$. Anche per questa stringa testuale, però, la possibilità che la tradizione veicolata dalla parafrasi del P.Oxy. XI 1404 si allinei piuttosto con quella degli Hermeneumata Pseudodositheana è supportata da elementi testuali e narrativi.

25 In questa prospettiva si confrontino Rodríguez Adrados 1999b, 9 e Pugliarello 2014, 83.

26 Della Corte 1966, 542.

27 Così Rodríguez Adrados 1999b, 9 e, più recentemente, Pugliarello 2014, 83. 
Dell'in aquam del papiro sono state proposte diverse interpretazioni: se, da un lato, Lenchantin de Gubernatis (1916, 199-201) non aveva esitato a metterlo in parallelo con l'altera (per alteram) della 1.4 e illustrarlo come un fenomeno di costruzione con l'accusativo per indicare lo stato in luogo, dall'altro, Della Corte $(1966,543)$ vi ha letto l'intenzione di rendere il senso del movimento indicato

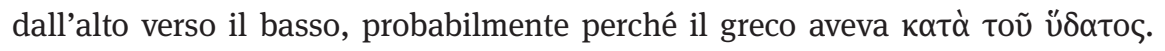
Che quest'ultima sia un'ipotesi maggiormente plausibile è provato dal fatto che

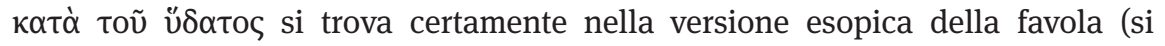
confrontino, infatti, Aesop. 185 Chambry $1960^{2}$, e, poi, Elio Teone). D’altra parte, l'uso di cum con il congiuntivo piuttosto che dell'indicativo è stato letto come un indice di miglior latino del parafraste ossirinchita rispetto a quello della favola approdata nella tradizione medievale degli Hermeneumata (Della Corte 1966, 544, dove si parla di una ripetizione videns vidit dello PseudoDositeo della quale le più recenti edizioni in circolazione non danno ragion d'essere).

Come si è detto, parlare del riflesso del pezzo di carne nell'acqua come di un'umbra è elemento lessicale - e, se si vuole, un'imprecisione alla quale si pone rimedio soltanto nei senari di Fedro, dove si parla piuttosto di simulacrum (1, 4, $3)^{28}$ - che allinea la tradizione del frammento ossirinchita con quella degli Hermeneumata Pseudodositheana e del Romulus, ma anche con tutta la precedente tradizione esopica in lingua greca.

1. 4: existima[v]it alteraくm>

Un richiamo a Phaedr. 1, 4, 4 (aliamque praedam ab alio cane ferri putans) è stato individuato in questa espressione del papiro ${ }^{29}$; non sfuggirà, però, che l'aestimans del Fragmentum Parisinum degli Hermeneumata Pseudodositheana è più vicino all'existimavit del papiro di quanto lo sia il putans di Fedro.

L'assenza della - $m$ nell'altera finale è stata spiegata come l'espressione della debolezza della consonante in fine di parola, diventata una risonanza nasale della vocale che la precedeva: «il suono debolissimo e quasi impercettibile di $m$ finale ci dà la ragione per cui il giovane ossirinchita, autore della parafrasi esopiana, assegnatagli forse come esercizio scolastico, essendo poco pratico dei segreti della grammatica e troppo fidente della guida infida, specialmente per uno straniero, della pronuncia, potesse scrivere in aquam invece di in aqua e altera in luogo di

28 Sulla questione si confrontino le osservazioni di Della Corte 1966, 544.

29 In questi termini si sono espressi Rodríguez Adrados 1999b, 9 e, in tempi più recenti, Pugliarello 2014, 83. 
alteram» (Lenchantin de Gubernatis 1916, 203) ${ }^{30}$. È questa un'ipotesi che non va accantonata dal momento che la debolezza (e l'omissione) della - $m$ finale è fenomeno ben noto e diffuso, indubbiamente marcato al livello di diacronia e diatopia (oltre che in diastratia), attestato spesso nei papiri latini d'Egitto (talora, copia di scribi ellenofoni che non avevano il latino per lingua madre $)^{31}$.

30 Basata su una ricostruzione difficilmente giustificabile è, invece, l'illustrazione di Della Corte 1966, 543-544, che ipotizza il nominativo sia riferibile piuttosto all'immagine di un altro cane - meglio, una cagna, così interpretata per errore del parafraste, indipendentemente dal fatto che la variante del sesso dell'animale può non essere scevra di ulteriore significato nelle due diverse tradizioni favolistiche e culturali.

31 Su questo fenomeno ci si limita qui a rinviare a Adams 2007, 128-132, dove si troveranno ulteriori riferimenti bibliografici sulla questione. 


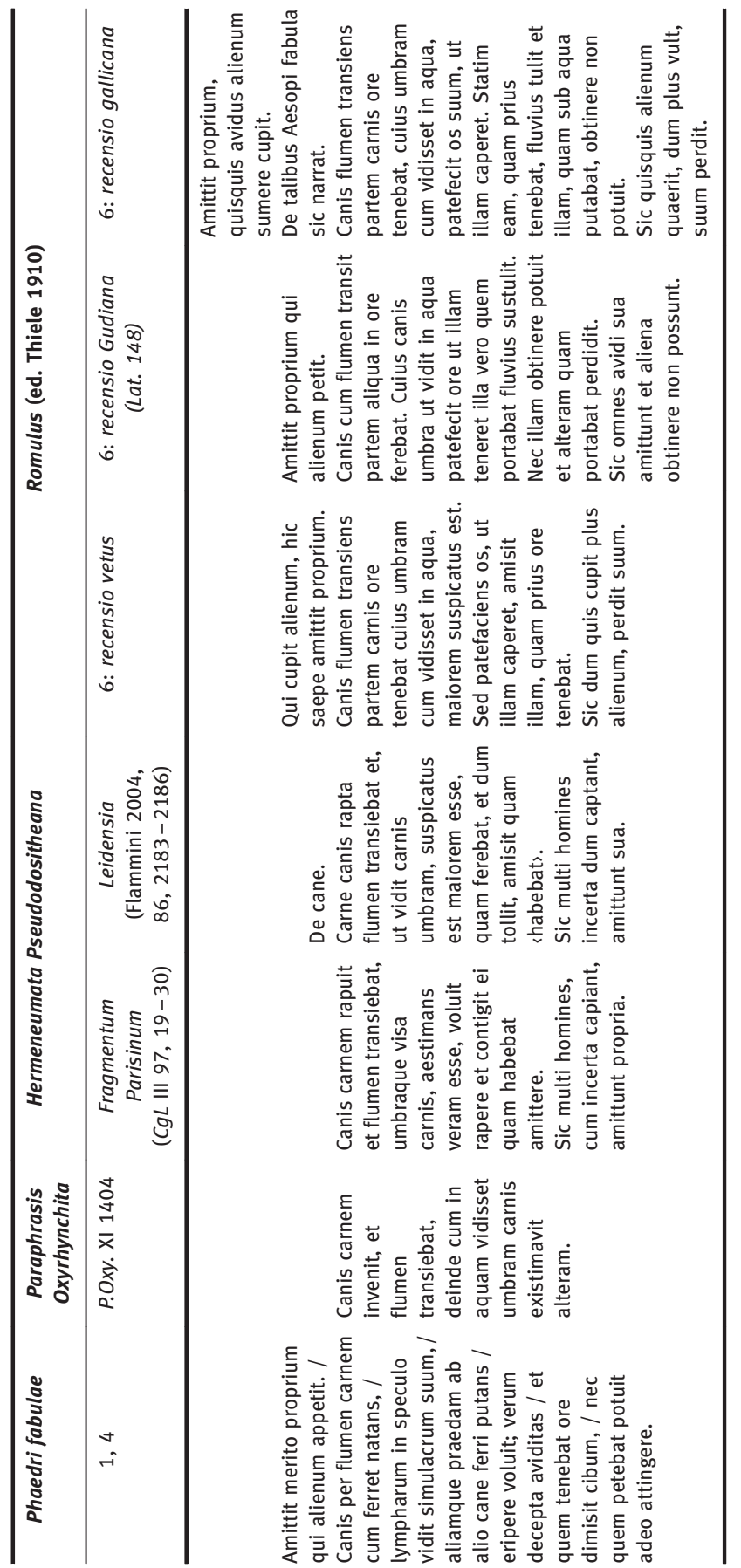

